\title{
5A2-Theory of the Optical Parametric Oscillator
}

\author{
A. YARIV, MEMBER, IEEE, AND W. H. LOUISELL, SENIOR MEMBER, IEEE
}

\begin{abstract}
A formalism for describing optical parametric oscillation is developed. The theory is applied to the derivation of the oscillation threshold condition, power output, the Manley-Rowe conditions, index matching, and frequency tuning.
\end{abstract}

\section{INTRODUCTION}

$\prod$ Nor $\mathrm{N}$ THIS PAPER, we present a general theory for parametric oscillation in the optical region. Since this phenomenon is the exact analog of parametric amplification and oscillation in the microwave region, its possibility was recognized a number of years ago [1]. The practical realization of optical parametric oscillation has been demonstrated by Giordmaine and Miller [2].

The experimental situation is as follows. A nonlinear optical crystal is placed within an optical resonator. A "pump" field at $\omega_{p}$ is then fed into the resonator. It is observed that at a certain pumping intensity, oscillation is set up simultaneously at frequencies $\omega_{1}$ and $\omega_{2}$, where $\omega_{1}+\omega_{2}=\omega_{p}$. These frequencies correspond to resonances of the optical structure or are near such resonances.

The theoretical approach takes the following form. The total electromagnetic field is expanded in a set of basis functions which consists of the proper field solutions of the optical resonator in the absence of any nonlinear elements. In this last case any one of the basis functions is a proper solution of Maxwell equations and, consequently, the coefficients of the expansion are time independent. When the nonlinearity is "turned on," the individual basis functions are no longer proper solutions and the coefficients must be taken as time dependent. In the limit of small coupling, this time dependence, for any one mode, contains the full information concerning the mode energy and its oscillation phase. The main concern of this paper is to obtain the solutions for these coefficients and, through them, follow the flow of power from the pump field into the $\omega_{1}$ and $\omega_{2}$ fields.

\section{The Electromagnetic Background}

The starting point is a solution of Maxwell equations inside a generalized resonator that contains a nonlinear dielectric material. The properties of the material are

Manuscript received June 20, 1966. Yariv was supported by the U. S. Air Force Laser Technology Group through Contract AF33(615)-2800. This paper was presented at the 1966 International Quantum Electronics Conference, Phoenix, Ariz.

A. Yariv is with the California Institute of Technology, Pasadena, Calif.

W. H. Louisell is with the Bell Telephone Laboratories, Inc., Murray Hill, N. J. characterized by a linear susceptibility $\chi^{L}$ and a susceptibility tensor of the third rank $d_{i i k}$. The induced polarization is given by

$$
P_{i} \epsilon_{0} \chi^{L} E_{i}+\sum_{i, k} d_{i j k} E_{i} E_{k}
$$

where, in general, the coefficients $d_{i j k}$ are functions of the frequencies of $P_{i}, E_{i}$, and $E_{k}$, respectively. If, however, the nonlinear medium is transparent in the region of interest, the coefficients $d_{i i k}$ are frequency independent. Another consequence is that all the $d_{i j k}$ elements which result from a mere rearrangement of the subscripts are equal [3].

Maxwell equations are

$$
\begin{aligned}
& \bar{\nabla} \times \bar{H}=\bar{\jmath}+\frac{\partial \bar{D}}{\partial t} \\
& \bar{\nabla} \times \bar{E}=-\frac{\partial \bar{B}}{\partial t}
\end{aligned}
$$

with the constitutive equations taken as

$$
\begin{aligned}
\bar{\jmath} & =\sigma \bar{E} \\
\bar{D} & =\epsilon_{0} \bar{E}+\bar{P}+\bar{P}^{\prime}
\end{aligned}
$$

where the total polarization is broken into the sum of the polarization $\bar{P}$, which is induced by the macroscopic fields and as given by (1), and an applied polarization $\bar{P}^{\prime}$, which represents the source feeding power into the resonator.

The electric and magnetic fields in the resonator can be expanded in terms of Slater's [4], [5] normal modes $\bar{H}_{a}$ and $\bar{E}_{a}$.

$$
\begin{aligned}
& \bar{H}(\bar{r}, t)=\sum_{a} \frac{\omega_{a} q_{n}(t)}{\sqrt{\mu_{0}}} \bar{H}_{a}(\bar{r}) \\
& \bar{E}(\bar{r}, t)=\sum_{a}-\frac{p_{a}(t)}{\sqrt{\epsilon}} \bar{E}_{a}(\bar{r}) .
\end{aligned}
$$

The vector functions $\bar{E}_{a}(\bar{r})$ and $\bar{H}_{a}(\bar{r})$ satisfy the relations

$$
\begin{aligned}
& \bar{\nabla} \times \bar{H}_{a}=k_{a} \bar{E}_{a} \quad k_{a}=\omega_{a} \sqrt{\mu_{0} \epsilon} \\
& \bar{\nabla} \times \bar{E}_{a}=k_{a} \bar{H}_{a}
\end{aligned}
$$

as well as $\bar{n} \times \bar{E}_{a}=0$ and $\bar{n} \cdot \bar{H}_{a}$ at the resonator walls. It follows from (6) and the two boundary conditions previously mentioned, that the sets $\bar{E}_{a}$ and $\bar{H}_{a}$ are orthogonal [4], [5]. We are free to choose their amplitudes so that they are normal. The orthonormality conditions are 


$$
\begin{aligned}
& \int_{V} \bar{E}_{a} \cdot \bar{E}_{b} d v=\delta_{a b} \\
& \int_{V} \bar{H}_{a} \cdot \bar{H}_{b} d v=\delta_{a b}
\end{aligned}
$$

where the integration extends over the whole resonator volume. Substituting (5) into (2) and taking the $i$ th component leads to

$$
\begin{aligned}
\sum_{a} \frac{\omega_{a} q_{a} k_{a}}{\sqrt{\mu_{0}}} E_{a i}= & -\sum_{a} \frac{\sigma}{\sqrt{\epsilon}} p_{a} E_{a i}-\epsilon_{0}\left(1+\chi^{L}\right) \sum_{a} \frac{\dot{p}_{a}}{\sqrt{\epsilon}} E_{a i} \\
& +\frac{\partial P_{i}^{\prime}}{\partial t}+\sum_{j, k, a, b} \frac{d_{i j k_{0}}}{\sqrt{\epsilon}} \frac{\partial}{\partial t}\left(p_{a} p_{b}\right) E_{a j} E_{b k}
\end{aligned}
$$

while from (3) and (5) we obtain

$$
-\sum_{a} \frac{p_{a} k_{a}}{\sqrt{\epsilon}} H_{a i}=-\mu_{0} \sum_{a} \frac{\omega_{a} \dot{q}_{a}}{\sqrt{\mu_{0}}} H_{a i}
$$

Next, we multiply each term in (8) by $E_{c i}$ and sum over $i(=1,2,3)$. Using $(7)$ and recalling that $\epsilon_{0}\left(1+\chi^{L}\right)=\epsilon$, we obtain

$$
\begin{aligned}
\frac{d p_{c}}{d t} & =-\omega_{c}^{2} q_{c}-\frac{\sigma}{\epsilon} p_{c}+\sum_{i, j, k, a, b} \frac{d_{i j \bar{k}}}{\epsilon^{3 / 2}} \\
& \cdot \int E_{c i} E_{a j} E_{b i} d v \frac{\partial}{\partial t}\left(p_{a} p_{b}\right)+\frac{1}{\sqrt{\epsilon}} \int \bar{E}_{c} \cdot \frac{\partial \bar{P}^{\prime}}{\partial t} d v .
\end{aligned}
$$

Multiplying (9) by $H_{c i}$ and summing gives

$$
\frac{d q_{0}}{d t}=p_{c} .
$$

Equations (10) and (11) are the equations of motion for the mode variables $p_{c}$ and $q_{c}$.

\section{Normal Modes}

Rather than carry out the analysis in terms of $p_{c}$ and $q_{c}$, we introduce the normal mode variables $a_{c}$ and $a_{c}^{*}$ which are defined as

$$
\begin{aligned}
a_{c} & =\frac{i}{\sqrt{2 \omega_{c}}}\left(p_{c}-i \omega_{c} q_{c}\right), \\
a_{c}^{*} & =\text { complex conjugate of } a_{c} .
\end{aligned}
$$

The expressions for $q_{c}$ and $p_{c}$ become

$$
q_{c}=\frac{1}{\sqrt{2} \omega_{c}}\left(a_{c}^{*}+a_{c}\right), \quad p_{c}=i \sqrt{\frac{\omega_{c}}{2}}\left(a_{c}^{*}-a_{c}\right) .
$$

The advantages of using the normal modes $a_{c}$ and $a_{c}^{*}$ rather than $p_{c}$ and $q_{c}$ have been discussed previously [5], [6].

The total energy stored in the electromagnetic field is

$$
\begin{aligned}
E & =\mu \int \bar{H} \cdot \bar{H} d v+\epsilon \int \vec{E} \cdot \vec{E} d v \\
& =\frac{1}{2} \sum_{c}\left(p_{c}^{2}+\omega_{c}^{2} q_{c}^{2}\right)
\end{aligned}
$$

where use has been made of (5) and (7). The energy $U_{0}$ in a single mode $\frac{1}{2}\left(p_{c}^{2}+\omega_{c}^{2} q_{c}^{2}\right)$ can also be expressed, using (13), as

$$
U_{c}=\frac{1}{2}\left(p_{c}^{2}+\omega_{c}^{2} q_{c}^{2}\right)=\omega_{c} a_{c}^{*} a_{c} .
$$

Since the energy of a photon is $\hbar \omega_{c}, a_{c}^{*} a_{c}$ is proportional to the number of photons in the mode $c$.

Using (13), we can transform the equations of motion (10) and (11) to a form containing the variables $a_{c}$ and $a_{c}^{*}$.

$$
\begin{aligned}
& \frac{d a_{c}}{d t}=-i \omega_{c} a_{c}+\frac{\omega_{c}}{2 Q_{c}}\left(a_{c}^{*}-a_{c}\right)-i \sum_{i, j, i, a, b} \sqrt{\frac{\omega_{a} \omega_{b}}{2 \omega_{c}}} \frac{d_{i j k}}{\epsilon^{3 / 2}} \\
& \cdot \int E_{c i} E_{a i} E_{b k} d v \frac{\partial}{\partial t}\left(a_{a}^{*}-a_{a}\right)\left(a_{b}^{*}-a_{b}\right) \\
&+\frac{i}{\sqrt{2 \omega_{c}}} \int \frac{\bar{E}_{c}}{\sqrt{\epsilon}} \cdot \frac{\partial \bar{P}^{\prime}}{\partial t} d v \\
& \frac{d a_{c}^{*}}{d t}=\left(\frac{d a_{c}}{d t}\right)^{*} .
\end{aligned}
$$

Next we define normal field variables $A_{c}(t)$ and $A_{c}^{*}(t)$ by

$$
a_{c}(t)=e^{-i \omega_{c} t} A_{c}(t), \quad a_{c}^{*}(t)=e^{i \omega_{c} t} A_{c}^{*}(t) .
$$

It is clear, from (16), that when $d_{i j k}=0$ and $\bar{P}^{\prime}=0$, $A_{c}(t)=$ const. $e^{-\left(\omega_{c} / 2 Q_{c}\right) t}$. We will assume that the coupling is sufficiently small so that

$$
\frac{d A_{c}}{d t} \ll \omega_{c} A_{c}
$$

for which case we may replace

$$
\frac{\partial}{\partial t}\left(a_{a}^{*}-a_{a}\right) \text { by } i \omega_{a}\left(a_{a}^{*}+a_{a}\right)
$$

and

$$
\frac{\partial}{\partial t}\left(a_{b}^{*}-a_{b}\right) \text { by } i \omega_{b}\left(a_{b}^{*}+a_{b}\right) \text {. }
$$

With these adiabatic approximations, the equations of motion (16) become

$$
\begin{aligned}
\frac{d a_{c}}{d t}= & -i \omega_{c} a_{c}-\frac{\omega_{c}}{2 Q_{c}}\left(a_{c}-a_{c}^{*}\right)+\sum_{i, j, k, a, b} \sqrt{\frac{\omega_{a} \omega_{b}}{2 \omega_{c}}} \frac{d_{i j k}}{\epsilon^{3 / 2}} \\
& \cdot \int E_{c i} E_{a j} E_{b i} d v\left[\omega_{b}\left(a_{a}^{*}-a_{a}\right)\left(a_{b}^{*}+a_{b}\right)\right. \\
& \left.+\omega_{a}\left(a_{a}^{*}+a_{a}\right)\left(a_{b}^{*}-a_{b}\right)\right]+\frac{i}{\sqrt{2 \omega_{c}}} \int \frac{\bar{E}_{c}}{\sqrt{\epsilon}} \cdot \frac{\partial \bar{P}^{\prime}}{\partial t} d v
\end{aligned}
$$

and

$$
\begin{aligned}
\frac{d a_{c}^{*}}{d t} & =i \omega_{c} a_{c}^{*}-\frac{\omega_{c}}{2 Q_{c}}\left(a_{c}^{*}-a_{c}\right)-\sum_{i, i, k, a, b} \sqrt{\frac{\omega_{a} \omega_{b}}{2 \omega_{c}}} \frac{d_{i j k}^{*}}{\epsilon^{3 / 2}} \\
& \cdot \int E_{c i} E_{a j} E_{b k} d v\left[\omega_{b}\left(a_{a}^{*}-a_{a}\right)\left(a_{b}^{*}+a_{b}\right)\right. \\
& \left.+\omega_{a}\left(a_{a}^{*}-a_{a}\right)\left(a_{b}^{*}-a_{b}\right)\right]-\frac{i}{\sqrt{2 \omega_{c}}} \int \frac{\bar{E}_{c}}{\sqrt{\epsilon}} \cdot \frac{\partial \bar{P}^{\prime}}{\partial t} d v
\end{aligned}
$$


where $\sigma / \epsilon=\omega_{c} / 2 Q_{c}$ is the decay rate for the field variables of mode $c$. These are the general working equations. They can be employed to obtain the differential equations which apply to special cases. In the next section we use them to obtain the equations describing the parametric oscillator.

\section{The Parametric Oscillator Equations}

Let us assume that the interactions are limited to three modes only, by means to be described shortly. Let these modes be denoted by the subscripts 1,2 , and $p$, and let their respective frequencies be $\omega_{1}, \omega_{2}$, and $\omega$. We shall refer to modes 1,2 , and $p$ as the signal, idler, and pump modes, respectively. Furthermore, we shall assume that mode $p$ (the pump) is driven by the polarization source so that

$$
\bar{P}^{\prime}(\tilde{r}, t)=\bar{P}^{\prime}(\bar{r}) \sin \omega t
$$

represents the applied polarization. If we consider the equation of motion for $a_{1}$, i.e., (20) with $c=1$, we notice that for the case of no coupling $\left(d_{i i k}=0\right)$, the solution is of the form $a_{1}(t)=a_{1}(0) e^{-i \omega_{2} t} e^{-\left(\omega_{2} / 2 Q_{1}\right) t}$. It follows that for the case of weak coupling the only cumulative contribution to $d a_{1} / d t$ comes from terms on the right side of (20) whose oscillatory factor is $e^{-i \omega_{1} t}$. The contributions from terms with frequencies considerably different from $\omega_{1}$ oscillate at a rate equal to the difference in frequencies, thus averaging out to zero in time spans that are long compared to the optical beat periods. If the free (i.e., no coupling) resonance frequencies satisfy the condition

$$
\omega=\omega_{1}+\omega_{2}
$$

then the product

$$
a_{2}^{*} a_{p}=A_{2}^{*}(t) A_{p}(t) e^{i\left(\omega_{2}-\omega\right) t}=A_{2}^{*}(t) A_{p}(t) e^{-i \omega_{2} t}
$$

on the right side of (20) satisfies the synchronism condition. Assuming that this happens for no other pair of modes, the equation for $a_{1}(t)$ can be written as

$$
\begin{aligned}
\frac{d a_{1}}{d t}=-i \omega_{1} a_{1}- & \frac{\omega_{1}}{2 Q_{1}} a_{1}+\sum_{i, i, k} \sqrt{\frac{\omega \omega_{2}}{2 \omega_{1}}} \frac{d_{i i k}}{\epsilon^{3 / 2}} \\
& \cdot \int E_{1 i} E_{2 i} E_{p k} d v 2\left(\omega-\omega_{2}\right) a_{2}^{*} a_{p}
\end{aligned}
$$

and similarly

$$
\begin{gathered}
\frac{d a_{2}^{*}}{d t}=i \omega_{2} a_{2}^{*}-\frac{\omega_{2}}{2 Q_{2}} a_{2}^{*}-\sum_{i, j, k} \sqrt{\frac{\omega \omega_{1}}{2 \omega_{2}}} \frac{d_{i j k}}{\epsilon^{3 / 2}} \\
\cdot \int E_{2 i} E_{1 j} E_{p l k} d v 2\left(-\omega+\omega_{1}\right) a_{1} a_{p}^{*} \\
\frac{d a_{p}}{d t}=-i \omega a_{p}-\frac{\omega}{2 Q_{p}} a_{p}+\sum_{i, j, k} \sqrt{\frac{\omega_{1} \omega_{2}}{2 \omega}} \frac{d_{i j k}}{\epsilon^{3 / 2}} \int E_{p i} E_{1 j} E_{2 k} d v \\
\cdot 2\left(-\omega_{1}-\omega_{2}\right) a_{1} a_{2}+\frac{i \sqrt{\omega}}{2 \sqrt{2 \epsilon}} \int \bar{E}_{p} \cdot \bar{P}_{p} d v e^{-i \omega t} \cdot \quad(24 \mathrm{c})
\end{gathered}
$$

Using (23), these equations are finally written as

$$
\begin{aligned}
\frac{d a_{1}}{d t}=-i \omega_{1} a_{1}-\frac{\omega_{1}}{2 Q_{1}} a_{1}+ & \sum_{i, i, k} \sqrt{2 \omega_{1} \omega_{1} \omega} \frac{d_{i j k}}{\epsilon^{3 / 2}} \\
& \cdot \int E_{1 i} E_{2 i} E_{p k} d v a_{2}^{*} a_{p}
\end{aligned}
$$

$$
\begin{aligned}
& \frac{d a_{2}^{*}}{d t}=i \omega_{2} a_{2}^{*}-\frac{\omega_{2}}{2 Q_{2}} a_{2}^{*} a_{2}^{*}+\sum_{i, j, k} \sqrt{2 \omega_{1} \omega_{2} \omega} \frac{d_{i j k}}{\epsilon^{3 / 2}} \\
& \cdot \int E_{2 i} E_{1 i} E_{p k} d v a_{1} a_{p}^{*} \\
& \frac{d a_{p}}{d t}=-i \omega a_{p}-\frac{\omega a_{p}}{2 Q_{p}}-\sum_{i, j, k} \sqrt{2 \omega_{1} \omega_{2} \omega} \frac{d_{i j k}}{\epsilon^{3 / 2}} \\
& \int E_{p i} E_{1 i} E_{2 k} d v a_{1} a_{2}+\frac{i \sqrt{\omega}}{2 \sqrt{2}} \int \frac{\bar{E}_{p} \cdot \bar{P}^{\prime}}{\sqrt{\epsilon}} d v e^{-i \omega t} .
\end{aligned}
$$

Equation (25) can be simplified further by taking advantage of the symmetry condition $d_{i j k}=d_{i i k}=d_{i k i}=$ $d_{k i i}=d_{i k i}=d_{k j i}$ which was previously discussed. Consider, for example, the summation in (25). If we interchange $i$ and $j$, we obtain

$$
\sum_{j, i, k} d_{i i k} \int E_{1 j} E_{2 i} E_{p l k} d v
$$

which is equal to the corresponding summation in (26) since $d_{i i k}=d_{i j k}$. The same argument can be used to show that the summation in (27) is equal to those of the first two. We can consequently define a single parameter $k$ as

$$
\kappa=\sum_{i, i, k} \sqrt{2 \omega_{1} \omega_{2} \omega} \frac{d_{i j k}}{\epsilon^{3 / 2}} \int E_{1 i} E_{2 j} E_{p k} d v
$$

to replace the triply-summed factors in (25). If, in addition, we define the pumping parameter $\lambda_{p}$ as

$$
\lambda_{p}=\frac{1}{2} \sqrt{\frac{\omega}{2 \epsilon}} \int \bar{E}_{p}(\vec{r}) \cdot \bar{P}^{\prime}(\vec{r}) d v,
$$

we can rewrite $(25),(26)$, and (27) as

$$
\begin{aligned}
& \frac{d a_{1}}{d t}=-i \omega_{1} a_{1}-\frac{\omega_{1}}{2 Q_{1}} a_{1}+\kappa a_{2}^{*} a_{p} \\
& \frac{d a_{2}^{*}}{d t}=i \omega_{2} a_{2}^{*}-\frac{\omega_{2}}{2 Q_{2}} a_{2}^{*}+\kappa a_{1} a_{p}^{*} \\
& \frac{d a_{p}}{d t}=-i \omega_{p} a_{p}-\frac{\omega}{2 Q_{p}} a_{p}-\kappa a_{1} a_{2}+i \lambda_{p} e^{-i \omega t}
\end{aligned}
$$

together with their complex conjugates.

In terms of the adiabatic variables $A_{i}(t)=a_{i}(t) e^{i \omega_{i} t}$, we have

$$
\begin{aligned}
& \frac{d A_{1}}{d t}=-\frac{\gamma_{1}}{2} A_{1}+\kappa A_{2}^{*} A_{p} \\
& \frac{d A_{2}^{*}}{d t}=-\frac{\gamma_{2}}{2} A_{2}^{*}+\kappa A_{1} A_{p}^{*} \\
& \frac{d A_{p}}{d t}=-\frac{\gamma_{p}}{2} A_{p}-\kappa A_{1} A_{2}+\imath \lambda_{p}
\end{aligned}
$$


and their complex conjugates. The decay rate $\gamma_{i}$ is defined as $\gamma_{i}=\omega_{j} / Q_{j}$, and is equal to the reciprocal decay time constant for the mode $j$. The $\gamma_{i}$ parameters account not so much for the losses in the medium as for the fact that the external coupling (i.e., reflector transmittances) is different for the three frequencies.

Equations (30) are the main result of the preceding section and constitute the starting point for the following analysis.

Before proceeding with the solutions of (30), it may be worthwhile to comment on the role of the pumping parameter $\lambda_{p}$. If $\lambda_{p}=0$, no steady-state oscillation can exist and the energy in the three modes decays with time.

As $\lambda_{p}$ is increased from zero, the steady-state solutions of (30) are $A_{1}=A_{2}=0$ and $A_{p}=2 i Q_{p} \lambda_{p} / \omega$. We shall refer to this region as "below threshold." In this region the pump field is proportional to the pump parameter $\lambda_{p}$, while the signal and idler modes are not excited.

By increasing $\lambda_{p}$ (and $A_{p}$ ) we reach a point at which a steady-state oscillation at $\omega_{1}$ and $\omega_{2}$ becomes possible. To find the necessary value of $A_{p}$, we set $d A_{1} / d t=d A_{2} / d t=0$ in (30). This gives

$$
\left|A_{p}\right|_{t h}^{2}=\frac{\omega_{1} \omega_{2}}{4 \kappa^{2} Q_{1} Q_{2}}=\frac{\gamma_{1} \gamma_{2}}{4 \kappa^{2}}
$$

for the threshold (start oscillation) value of $\left|A_{p}\right|^{2}$. Exactly at threshold $A_{1}=A_{2}=0$, so that from the last equation of (30) (with $d A_{p} / d t=0$ ) and (31), we obtain

$$
\left|\lambda_{p}\right|_{t h}^{2}=\frac{\gamma_{1} \gamma_{2} \gamma_{p}^{2}}{16 \kappa^{2}}
$$

for the (squared) pumping parameter at threshold.

As the pumping is increased above the threshold value as given by (32), $A_{p}$ can no longer increase with it. This is due to the fact that no steady-state solution for $A_{1}$ and $A_{2}$ is possible if $\left|A_{v}\right|^{2}$ exceeds its threshold value as given by (31). The last equation of (30) shows that this "clamping" of $A_{p}$ in the face of an increasing $\lambda_{p}$ is only possible if the product $\kappa A_{1} A_{2}$ "picks up the slack." The increase in pumping above threshold is thus seen to give rise to an oscillation at $\omega_{1}$ and $\omega_{2}$, with no further increase in the intensity of the pump mode. This is the equivalent of gain saturation in a laser oscillator, where the population inversion, and hence, the gain, is proportional to the pumping rate below threshold but "saturates" once threshold is exceeded. Further increases in the pumping power give rise to higher oscillation (and power output) level.

It is evident that a description of the saturation (above threshold) behavior of the parametric optical oscillator depends on the use of separate parameters for describing the pump field $\left(A_{p}\right)$ and the pumping intensity $\left(\lambda_{p}\right)$. Analyses which employ only $A_{D}$ (or some suitable equivalent thereof) can still be used to derive the threshold conditions. This has been done by a number of authors.

\section{The Power Relations}

From (30) we can obtain explicit expressions for the power output at the signal frequency $\omega_{1}$ and the idler $\omega_{2}$. Since the stored energy in the $i$ th mode is $\omega_{i} a_{i} a_{i}^{*}=\omega_{i} A_{i} A_{i}^{*}$, the total power output is $P_{i}=\omega_{i} A_{i} A_{i}^{*}\left(\omega_{i} / Q_{i}\right)$, or using $\gamma_{i}=\omega_{i} / Q_{i}$

$$
P_{i}=\gamma_{i} \omega_{i} A_{i}^{*} A_{i} .
$$

From the last equation of (30), with $d / d t=0$, we obtain

$$
-\frac{\gamma_{p}}{2} A_{p}-\kappa A_{1} A_{2}+i \lambda_{p}=0
$$

and multiplying the first equation of (30) with $d / d t=0$ by $A_{2}$,

$$
\frac{\gamma_{1}}{2} A_{1} A_{2}=\kappa\left|A_{2}\right|^{2} A_{p}
$$

Combining the last two equations leads to

$$
A_{p}=\frac{2 i \lambda_{p}}{\gamma_{p}+\frac{4 \kappa^{2}}{\gamma_{1}}\left|A_{2}\right|^{2}} .
$$

Repeating this procedure with the second equation of (30) gives

$$
A_{p}^{*}=\frac{-2 i \lambda_{p}}{\gamma_{p}+\frac{4 \kappa^{2}}{\gamma_{2}}\left|A_{1}\right|^{2}} .
$$

Equations (38) and (37) are compatible only if $\gamma_{1}\left|A_{1}\right|^{2}=$ $\gamma_{2}\left|A_{2}\right|^{2}$, or, using (34), when

$$
\frac{P_{1}}{\omega_{1}}=\frac{P_{2}}{\omega_{2}}
$$

so that the number per second of photons generated at $\omega_{1}$ is equal to that generated at $\omega_{2}$. This is true regardless of the respective losses. The losses do, however, determine the magnitude of the power at $\omega_{1}$ (and $\omega_{2}$ ). Equations (39) were first derived by Manley and Rowe [7]. Viewed from the quantum mechanical point of view, (39) reflects the fact that the parametric process can be described as a scattering event in which a pump photon of energy $\hbar \omega$ is "annihilated" while, simultaneously, two photons-one at $\omega_{1}$, the other at $\omega_{2}$-are created. Conservation of energy dictates that $\omega=\omega_{1}+\omega_{2}$, while the basic nature of the scattering guarantees that the number of photons created or annihilated at each of the three frequencies are equal [8].

To obtain an expression for $P_{1}$, we equate the value of $A_{p} A_{p}^{*}$ as given by (38) to the threshold value (31). The resulting equality is then solved for $A_{1} A_{1}^{*}$ with the result

$$
\frac{P_{1}}{\omega_{1}}=\gamma_{1}\left|A_{1}\right|^{2}=\sqrt{\gamma_{1} \gamma_{2}} \frac{\lambda_{p}}{\kappa}-\frac{\gamma_{1} \gamma_{2} \gamma_{p}}{4 \kappa}
$$

which, of course, is only valid when $\sqrt{\gamma_{1} \gamma_{2}} \lambda_{p} / \kappa \geq \gamma_{1} \gamma_{2} \gamma_{p} / 4 \kappa^{2}$, i.e., above threshold. 
Using (32) for $\lambda_{p}$ at threshold we can rewrite (40) as

$$
\begin{aligned}
\frac{P_{1}}{\omega_{1}} & =\frac{\sqrt{\gamma_{1} \gamma_{2}}}{\kappa}\left(\lambda_{p}\right)_{t h}\left[\frac{\lambda_{p}}{\left(\lambda_{p}\right)_{t h}}-1\right] \\
& =\frac{\gamma_{1} \gamma_{2} \gamma_{p}}{4 \kappa^{2}}\left[\frac{\lambda_{p}}{\left(\lambda_{p}\right)_{t h}}-1\right] .
\end{aligned}
$$

The factor $\gamma_{1} \gamma_{2} / 4 \kappa^{2}$ is, according to (31), the value of $\left|A_{p}\right|^{2}$ at threshold, so that (41) can be written as

$$
\frac{P_{1}}{\omega_{1}}=\frac{P_{2}}{\omega_{2}}=\frac{\left(P_{p}\right)_{t h}}{\omega_{p}}\left[\frac{\lambda_{p}}{\left(\lambda_{p}\right)_{t h}}-1\right] .
$$

Equation (42) is the basic power equation. $\left(P_{p}\right)_{t h} / \omega_{n}$ is the rate of photon dissipation at threshold at $\omega_{p} . \lambda_{p} /\left(\lambda_{p}\right)_{t h}$, the pumping factor, is the factor by which threshold is exceeded. According to (42), each time the pump intensity is exceeded by an amount equal to the threshold intensity, the photon output at $\omega_{1}$ and $\omega_{2}$ is increased by $\left(P_{p}\right)_{l h} / \omega_{p}$. This linear power relationship is shown in Fig. 1, in which the $\omega_{1}$ (and $\omega_{i}$ ) photon output, relative to the pump photon at threshold, is plotted vs. the pumping intensity relative to its threshold value.

The conservation of power takes the form of

$$
\left(P_{p}\right)_{i h}+P_{1}+P_{2}=\left(P_{p}\right)_{t h} \frac{\lambda_{p}}{\left(\lambda_{p}\right)_{t h}}=P_{p}
$$

which follows from (42) and the relation $\omega_{p}=\omega_{1}+\omega_{2}$. This relation shows that the total power input is accounted for by the outputs at $\omega_{1}, \omega_{2}$, and the dissipation at $\omega_{p}$, which is "clamped" at its threshold value $\left(P_{p}\right)_{t h}$.

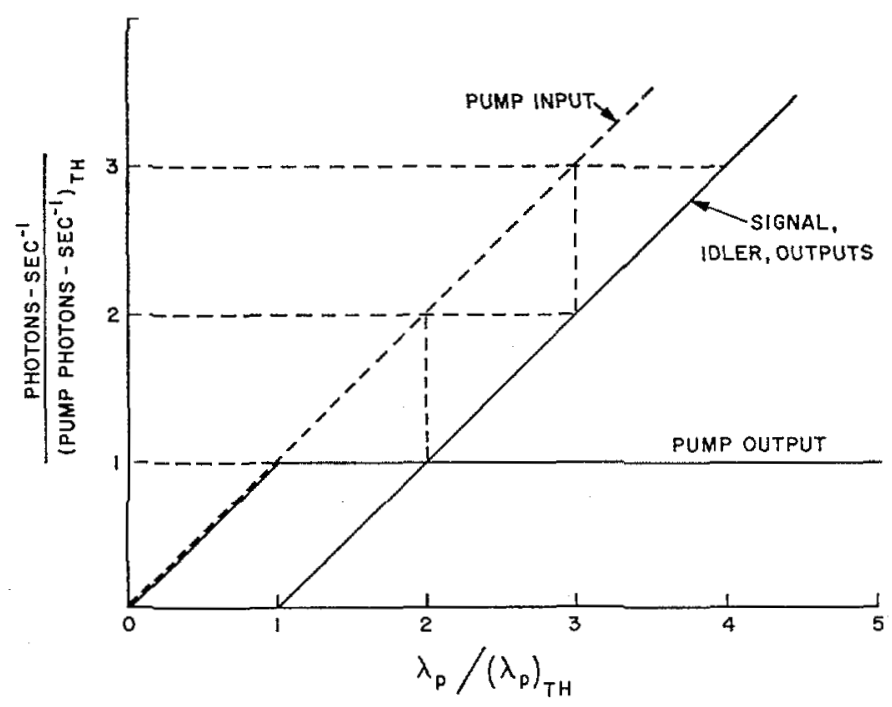

Fig. 1. The power output (in photons per second) at $\omega_{1}$ and $\omega_{2}$ relative to the threshold pump input. The linear relation shows how, above threshold, the excess pumping power at $\omega$ is converted into oscillation power at $\omega_{1}$ and $\omega_{2}$, where $\omega=\omega_{1}+\omega_{2}$. The horizontal portion of the pump output curve corresponds to the saturation of the pump field intensity inside the resonator above threshold.

\section{Optrmum Coupling}

Having obtained in the last section explicit expressions for the power output as a function of the loss, coupling; and pumping parameters, we are in a position to solve for the optimum coupling condition which maximizes the power output.

The starting point is (41),

$$
\frac{P_{1}}{\omega_{1}}=\sqrt{\gamma_{1} \gamma_{2}} \frac{\lambda_{p}}{\kappa}-\frac{\gamma_{1} \gamma_{2} \gamma_{p}}{4 \kappa^{2}} .
$$

The loss parameters $\gamma_{1,2}$ are related to resonator Q's by

$$
\gamma_{1,2}=\frac{\omega_{1,2}}{Q_{1,2}}=\frac{1}{\tau_{1,2}}=\frac{c\left(1-R_{1,2}\right)}{2 L_{1,2}}
$$

where $R_{1,2}$ are the effective ${ }^{1}$ reflectances and $L_{1,2}$ is the resonator length at $\omega_{1,2}, \gamma_{1,2}$ can be expressed as the sum of two terms. The first represents the unaviodable residual losses and is taken as $\left(\gamma_{1,2}\right)_{i}$. The second term is due to the useful output coupling of power and is taken as $\left(\gamma_{1,2}\right)_{e}$.

$$
\gamma_{1,2}=\left(\gamma_{1,2}\right)_{i}+\left(\gamma_{1,2}\right)_{\circ}
$$

In analogy with the laser coupling analysis [9], we define the ratio of the power output to the residual losses as

$$
S_{1,2}=\frac{\left(\gamma_{1,2}\right)_{e}}{\left(\gamma_{1,2}\right)_{i}}
$$

and refer to it as the coupling parameter. In practice we can vary $S_{1,2}$ by varying the reflector transmittance.

We first consider the case when $\gamma_{1}==\gamma_{2}$. This may happen when $\omega_{1}$ and $\omega_{2}$ are so near to each other that separate control of the reflectances is not practical. When this is the case, (41) becomes

$$
\frac{P_{1}}{\omega_{1}}=\frac{\gamma \lambda_{p}}{\kappa}-\frac{\gamma^{2} \gamma_{p}}{4 \kappa^{2}}
$$

or, using (44) and (45),

$$
\frac{P_{1}}{\omega_{1}}=\gamma_{i}(S+1) \frac{\lambda_{p}}{\kappa}-\frac{\gamma_{i}^{2} \gamma_{p}}{4 \kappa^{2}}(S+1)^{2} .
$$

The part of $P_{1}$ which is available as output is $\left(P_{1}\right)_{e}$ and is given by

$$
\frac{\left\langle P_{1}\right\rangle_{e}}{\omega_{1}}=\frac{P_{1}}{\omega_{1}} \frac{S}{S+1}
$$

which, using (46), becomes

$$
\frac{\left(P_{1}\right)_{e}}{\omega_{1}}=\frac{\gamma_{i} \lambda_{p}}{\kappa} S-\frac{\gamma_{i}^{2} \gamma_{p}}{4 \kappa^{2}}(S+1) S .
$$

Maximization of the power output $\left(P_{1}\right)_{\text {. with respect to }}$ $S$ yields

$$
S_{o p t}=\frac{1}{2}\left(\frac{4 \kappa \lambda_{p}}{\gamma_{i} \gamma_{p}}-1\right)
$$

\footnotetext{
1 i.e., adjusted so as to account for the total loss per pass.
} 
According to (32), the factor $\gamma_{i} \gamma_{p} / 4 \kappa$ is equal to the value of the threshold pumping parameter $\left(\lambda_{p}\right)_{t h}$ for zero coupling (i.e., for $\left.\gamma_{1}=\gamma_{2}=\gamma_{i}\right)$. We designate it as $\left(\lambda_{p}\right)_{t h}^{0}$, and rewrite the expression for the optimum coupling as

$$
S_{\mathrm{opt}}=\frac{1}{2}\left[\frac{\lambda_{p}}{\left(\lambda_{p}\right)_{t h}^{6}}-1\right]
$$

which shows that near threshold the optimum coupling is near zero, since even a slight increase in coupling, and hence, in losses, quenches the oscillation.

\section{The Threshold Condition}

The threshold condition as reproduced from (31), is

$$
\left|A_{p}\right|^{2}=\frac{\gamma_{1} \gamma_{2}}{4 \kappa}
$$

where $\kappa$, the coupling parameter, is defined by (28), and involves an integral over the volume of the resonator of the product of the electric field components at $\omega_{1}, \omega_{2}$, and $\omega$. Let us consider a resonator supporting plane wave-like propagation along the $x$-direction. Let the length (in the $x$ direction) be $L$ and the beam cross sections $A$. The spatial variation of the normalized field amplitudes is then

$$
E_{a}(r)=\sqrt{\frac{2}{L A}} \sin \frac{\pi a}{L} x
$$

the form of which satisfies (7).

The parameter $A_{x}$ can be replaced by an expression the form of which satisfies (7).

$$
E_{p}(x)=E_{p 0} \sin \frac{\pi m x}{L}
$$

by recognizing that the stored energy at $\omega, \omega\left|A_{p}\right|^{2}$, is equal, according to (52), to $\frac{1}{4} \epsilon E_{p 0}^{2} V$, where $V=L A$ is the resonator mode volume. This gives $\left|A_{p}\right|^{2}=(\epsilon / 4 \omega) E_{p 0}^{2} V$, and the threshold condition (50) becomes

$$
\left(E_{p 0}^{2}\right)_{t h}=\frac{\omega \gamma_{1} \gamma_{2}}{\kappa^{2} \epsilon V} .
$$

To evaluate $\kappa$ we first need to obtain an expression for the integral of (28). Taking $E_{1 i}, E_{2 j}, E_{p k}$ in the form of (51),

$$
\begin{aligned}
& E_{1 i}=\sqrt{\frac{2}{L A}} \sin k_{1} x \\
& E_{2 i}=\sqrt{\frac{2}{L A}} \sin k_{2} x \\
& E_{p k}=\sqrt{\frac{2}{L A}} \sin k x
\end{aligned}
$$

and defining

$$
\Delta k=k-\left(k_{1}+k_{2}\right)
$$

we obtain from (28)

$$
\kappa=\frac{1}{2} \sqrt{\omega \omega_{1} \omega_{2}} \frac{d_{i j k}}{\epsilon^{3 / 2} V^{1 / 2}} F(\Delta k L)
$$

where

$$
F(\Delta k L)=4 \frac{\sin ^{2}\left(\frac{\Delta k L}{2}\right)}{\Delta k L} .
$$

Substituting (56) into (53) leads to the following form of the threshold condition:

$$
\frac{d_{i j k}\left(E_{p 0}\right)_{t h}}{2 \epsilon} F(\Delta k L)=\frac{1}{\sqrt{Q_{1} Q_{2}}}
$$

where we used $\gamma_{1,2}=\omega_{1,2} / Q_{1,2}$. This form is the most convenient for numerical calculations. $F(\Delta k L)$ has, as will be shown, a maximum magnitude of near unity. $d_{i j k} E_{p 0}$ is equal to $\delta \epsilon$, the amplitude of the modulation (at $\omega$ ) of the optical dielectric constant $\epsilon$ by the pump field. Equation (58) is thus of the form

$$
\delta \epsilon / 2 \epsilon=\frac{1}{\sqrt{Q_{1} Q_{2}}},
$$

which is similar to the oscillation condition of varactor paramps, which is

$$
\frac{\Delta c}{2 \sqrt{c_{1} c_{2}}}=\frac{1}{\sqrt{Q_{1} Q_{2}}}
$$

where the time-varying coupling capacitance is

$$
c=c_{0}+\Delta c \cos \omega t
$$

\section{Index Matching and Frequency Tuning}

According to (58), the threshold pump field $\left(E_{p 0}\right)_{t h}$ is inversely proportional to $F(\Delta k L)$, as defined by (57). The maximum of $F(\Delta k L)$ occurs for $\Delta k L=2.33$ and has a value of $F(2.33)=1.445$. The index matching condition for plane waves is $\Delta k=0[10]$. For practical purposes, the distinction between the two cases is not significant, since for $\lambda \sim 1 \mu,|k| \sim 10^{5}$.

According to $(58)$, the pair of frequencies $\omega_{1}$ and $\omega_{2}$, which is excited into oscillation, is that for which the factor $\sqrt{Q_{1} Q_{2}} F(\Delta k L)$ is a maximum. The maximum of $F(\Delta k L)$ was shown to occur, very nearly, for

$$
k=k_{1}+k_{2}
$$

so that, for frequency independent $Q$ 's, the oscillating frequencies $\omega_{1}, \omega_{2}$ will be those for which condition (59) and the energy conservation condition $\omega=\omega_{1}+\omega_{2}$ are satisfied. For a given orientation of the nonlinear crystal with respect to the axis of the optical resonator, (59) will be satisfied for only one $\omega_{1}, \omega_{2}$ pair. ${ }^{2}$ The orientation of the nonlinear crystal will thus provide a means for tuning the output frequencies.

As an example, we consider the case of a $\mathrm{KDP}\left(\mathrm{KH}_{2} \mathrm{PO}_{4}\right)$ crystal. When $\omega_{1}=\omega_{2}$, i.e., the degenerate oscillator, the

\footnotetext{
2 Under certain conditions in some crystals it is possible to satisfy (59) for two different pairs of frequencies.
} 
index matching condition (59), becomes $k=2 k_{1}$. By choosing $\omega$ as an extraordinary ray and $\omega_{1}=\omega_{2}$ as an ordinary ray, this condition is the same as that of maximum second harmonic generation [10], $\left(\omega=2 \omega_{1}\right)$. This is done by selecting the direction of propagation in the crystal to be at an angle $\theta_{m}$ with respect to the optic axis where [10]

$$
\sin ^{2} \theta_{m}=\frac{\left(\frac{1}{n_{0}^{\omega_{2}}}\right)^{2}-\left(\frac{1}{n_{0}^{2 \omega_{1}}}\right)^{2}}{\left(\frac{1}{n_{e}^{2 \omega_{1}}}\right)^{2}-\left(\frac{1}{n_{0}^{2 \omega_{1}}}\right)^{2}}
$$

where $n_{e}^{2 \omega_{1}}$, for example, is the extraordinary index of refraction at $2 \omega_{1}$.

When $\omega_{1} \neq \omega_{2}$, we can still satisfy (59) with the $\omega_{1}$ and $\omega_{2}$ taken as ordinary rays while the pump at $\omega$ is an extraordinary ray. For this case, (59) becomes

$$
n_{e}^{\omega}(\theta)=\frac{\omega_{1}}{\omega} n_{0}^{\omega_{1}}+\frac{\omega_{2}}{\omega} n_{0}^{\omega_{2}}
$$

It is convenient to introduce a parameter $\Delta$, which is a measure of the frequency deviation from the degenerate condition

$$
\begin{aligned}
& \omega_{1}=\frac{\omega}{2}(1+\Delta) \\
& \omega_{2}=\frac{\omega}{2}(1-\Delta) .
\end{aligned}
$$

We can, likewise, define $\gamma$ by $n_{0}^{\omega_{1}}=n_{0}^{\omega_{2}}(1+\gamma)$. Using the relation

$$
\left(\frac{1}{n_{e}^{\omega}(\theta)}\right)^{2}=\left(\frac{1}{n_{0}^{\omega}}\right)^{2} \cos ^{2} \theta+\left(\frac{1}{n_{e}^{\omega}}\right) \sin ^{2} \theta
$$

in (61), we obtain the following expression for the index matching angle $\theta$, for the triplet $\omega, \omega_{1}, \omega_{2}$.

$$
\sin ^{2} \theta=\frac{\left(\frac{1}{1+\frac{\gamma}{2}+\frac{\Delta \gamma}{2}}\right)^{2}\left(\frac{1}{n_{0}^{\omega_{2}}}\right)^{2}-\left(\frac{1}{n_{0}^{\omega}}\right)^{2}}{\left(\frac{1}{n_{e}^{\omega}}\right)^{2}-\left(\frac{1}{n_{0}^{\omega}}\right)^{2}} .
$$

Next we assume that near $\omega / 2$ we can approximate the dependence of $n_{0}$ on the frequency $\omega^{\prime}$ by $n_{0}\left(\omega^{\prime}\right)=n_{0}\left(\frac{\omega}{2}\right)+a\left(\omega^{\prime}-\frac{\omega}{2}\right)+b\left(\omega^{\prime}-\frac{\omega}{2}\right)^{2}+c\left(\omega^{\prime}-\frac{\omega}{2}\right)^{3}$

where $a, b$, and $c$ are constants which can be determined from the dispersion data. Using (63), we have

$$
\begin{aligned}
& n_{0}^{\omega_{1}}=n_{0}\left(\frac{\omega}{2}\right)+a \frac{\omega \Delta}{2}+\frac{b}{4} \omega^{2} \Delta^{2}+\cdots \\
& n_{0}^{\omega_{\mathrm{a}}}=n_{0}\left(\frac{\omega}{2}\right)-a \frac{\omega \Delta}{2}+\frac{b}{4} \omega^{2} \Delta^{2}+\cdots .
\end{aligned}
$$

Next, we can obtain an expression for $\gamma=n_{0}^{\omega_{2}} / n_{0}^{\omega_{2}}-1$ in terms of $a, b$, and $\Delta$. Substituting this expression into (62), and going through some quite tedious algebra, we obtain

$\sin 2 \theta_{m} \Delta \theta=\frac{\left(\frac{1}{n_{0}^{\omega / 2}}\right)^{2}\left[-\frac{5}{4} \frac{a^{2} \omega^{2}}{\left(n_{0}^{\omega / 2}\right)^{2}}-\frac{a \omega}{n_{0}^{\omega / 2}}+\frac{b \omega^{2}}{2 n_{0}^{\omega / 2}}\right] \Delta^{2}}{\left(\frac{1}{n_{e}^{\omega}}\right)^{2}-\left(\frac{1}{n_{0}^{\omega}}\right)^{2}}$

where $\Delta \theta=\theta-\theta_{m}$ is the deviation of the index-matching angle (for the $\omega, \omega_{1}, \omega_{2}$ triplet) from the degenerate index matching angle $\theta_{m}$. In deriving (65), we keep only terms up to, but not including, $\Delta^{4}$.

Equation (65) provides a direct relation between the angular position $\Delta \theta$ and the frequency parameter $\Delta$; i.e., it is the $\omega_{1}$ vs. $\theta$ (or $\omega_{2}$ vs. $\theta$ ) tuning curve. Similar expressions can be obtained for other crystals and different polarization choices for $\omega_{1}, \omega_{2}$, and $\omega$.

\section{REFERENCES}

[1] R. H. Kingston, "Parametric amplification and oscillation at optical frequencies," Proc. IRE (Correspondence), vol. 50, p. 472, April 1962.

[2] J. A. Giordmaine and R. C. Miller, Phys. Rev. Letters, vol. 14, p. $973,1965$.

[3] D. A. Kleinman, Phys. Rev., vol. 126, p. 1977, 1962.

4] J. C. Slater, Microwave Electromics. Princeton, N. J.: Van Nostrand, 1950 , p. 57.

[5] A. Yariv, "Parametric interactions of optical modes," IEEE J. of Quantum Electronics, vol. QE-2, pp. 30-37, February 1966.

[6] A. Yariv, "Quantum theory for parametric interactions of light and hypersound," IEEE J. of Quantum Electronics, vol. QE-1, pp. 28-36, April 1965

[7] J. M. Manley and H. E. Rowe, "General energy. relations in nonlinear reactances," Proc. IRE (Correspondence), vol. 47, pp. 2115-2116, December 1959 .

[8] W. H. Louisell, A. Yariv, and A. E. Siegman, Phys. Rev., vol. 124, p. 1646, 1961.

[9] A. Yariv and J. P. Gordon, "The laser," Proc. IEEE, vol. 51, pp. 4-29, January 1963.

[10] J. A. Giordmaine, Phys. Rev. Letters, vol. 8, p. 19, 1962. 